\title{
RELACIÓN ENTRE VARIABLES NEUROCOGNITIVAS Y PERSONALIDAD EN DELINCUENTES RELATIONSHIP BETWEEN NEUROCOGNITIVE VARIABLES AND PERSONALITY IN OFFENDERS
}

\author{
Juan Antonio Becerra García y Ana García León \\ Universidad de Jaén. jbecerra@ujaen.es
}

Cómo referenciar este artículo/How to reference this article:

Becerra García, J. y García León, A. (2014). Relación entre Variables Neurocognitivas y Personalidad en Delincuentes [Relationship between Neurocognitive Variables and Personality in Offenders]. Acción Psicológica, 11(2), 117-126. http://dx.doi.org/10.5944/ap.11.2.10857

\begin{abstract}
Resumen
Recientes estudios muestran que rasgos de personalidad del Modelo de los Cinco Factores y funciones neurocognitivas frontales comparten una misma base neurobiológica. En población penitenciaria las relaciones entre estas funciones y rasgos ha sido poco explorada. Este estudio pretende examinar la relación entre personalidad y funciones neurocognitivas en delincuentes y determinar si las medidas de funcionamiento neurocognitivo frontal contribuyen a predecir algún porcentaje de varianza en rasgos de personalidad de muestras penitenciarias. Noventa y nueve hombres (distribuidos en grupos: agresores sexuales de adultos, delincuentes no sexuales y grupo control) realizaron el Inventario de Personalidad NEO de Cinco Factores (NEO-FFI) y el Trail Making Test (TMT, partes A y B). La ejecución en la parte A del TMT se relaciono con el nivel de Extraversión de los delincuentes sexuales y con el nivel de Neuroticismo de delincuentes no sexuales y explica un porcentaje significati-
\end{abstract}

vo de la varianza de estos dominios de personalidad en ambos grupos. En conclusión, la relación entre el funcionamiento neurocognitivo y la personalidad parece ser diferente en función del tipo de delito cometido, aunque los resultados deben ser interpretados de forma preliminar.

Palabras clave: Personalidad; neuropsicología; delincuentes; velocidad de procesamiento.

Abstract
Recent studies show that traits of Five-Factor
Model of personality and frontal neurocogniti-
ve functions share a common neurobiological
basis. In penitentiary population, the relation-
ships between these functions and traits have
been little studied. This study aims to examine
the relationship between personality and neu-
rocognitive functions in offenders and deter-
mine whether measures of frontal neurocogni-
tive functioning help to predict some
percentage of variance in personality traits of


penitentiary samples. Ninety-nine men (divided into groups: sex offenders against adults, non-sex offenders and control group) performed the NEO Five-Factor Personality Inventory (NEO-FFI) and Trail Making Test (TMT; parts $\mathrm{A}$ and $\mathrm{B})$. The performance in part $\mathrm{A}$ of TMT was associated with Extraversion level on sex offenders and with Neuroticism level on non-sexual offenders, and explained a significant proportion of the variance of these domains personality in both groups. In conclusion, the relationship between neurocognitive functioning and personality seems to be different depending on the type of crime committed, although findings must be interpreted in a preliminary way.

Key words: Personality; neuropsychology; offenders; processing speed.

\section{Introducción}

El Modelo de los Cinco Factores (MCF) organiza la personalidad en cinco amplias dimensiones: Neuroticismo (N), Extraversión (E), Apertura (AP), Amabilidad (AM) y Responsabilidad (R) (Costa y McCrae, 1992; McCrae y Costa, 1997). El N es una dimensión general de afecto negativo. La E se caracteriza por la tendencia a buscar actividades estimulantes y compañía social. La AP refleja la mayor sensibilidad estética y emocional, la preferencia por la novedad y la curiosidad intelectual. La AM sería el dominio que caracterizaría a personas cooperativas, generosas y simpáticas. Por último, la $\mathrm{R}$ representaría la tendencia a mostrar autodisciplina y actuar de forma leal y orientada al logro (Costa y McCrae, 1992; McCrae y Costa, 1997). Este modelo dimensional proporciona un marco teórico robusto sobre las dimensiones básicas que subyacen a la personalidad humana a lo largo de la edad, el género o la cultura (McCrae y Costa, 1997).

Las investigaciones más recientes en relación al MCF se centran en el estudio de las bases neurales subyacentes a las diferentes dimensiones que lo conforman. Estas investigaciones muestran que todos los dominios de personalidad del modelo se relacionan con diferentes estructuras y circuitos del cortex frontal. Así el $\mathrm{N}$ se relaciona negativamente con una conectividad y funcionamiento efectivo (procesamiento, integración, ejecución, etc.) entre el cortex prefrontal y la amígdala (Cremers et al., 2010). La E se relaciona con el volumen del cortex prefrontal (De Young et al., 2010) y con el volumen de fibras que conectan estas áreas con el cortex orbitofrontal y motor (Cohen, Schoene-Bake, Elger y Weber, 2009). Por su parte el rasgo AP está relacionado con el rendimiento en tareas cognitivas (como flexibilidad cognitiva) que implican al cortex prefrontal dorsolateral (Schretlen, van der Hulst, Pearlson y Gordon, 2010) y con el volumen de fibras que conectan áreas frontales con áreas temporo-parietales (Takeuchi et al., 2010). La AM se ha relacionado con tareas cognitivas relacionadas con el funcionamiento de áreas frontales (Nettle y Liddle, 2008) y con el volumen de fibras de conexión de estructuras frontales con áreas corticales (parietales) y subcorticales (núcleo estriado) (Charlton, Barrick, Markus y Morris, 2009). Por último, el dominio $\mathrm{R}$ se ha relacionado positivamente con el volumen de áreas prefrontales y orbitofrontales (De Young et al. 2010; Jackson, Balota y Head, 2011). Recientemente, Xu y Potenza (2012) mejoran las limitaciones de los estudios anteriores y concluyen que las fibras de conexión (encargadas de unir áreas corticales prefrontales con otras zonas corticales y subcorticales) son las áreas neurales más relacionadas con los cinco dominios de personalidad del modelo. Estas fibras de conexión son las responsables de la velocidad de conducción nerviosa y se han relacionado principalmente con la velocidad de procesamiento viso-motora y cognitiva (capacidad neurocognitiva básica para realizar funciones cognitivas superiores) como muestran diferentes trabajos (Delano-Wood et al., 2008; Turken et al., 2008).

Además de estar relacionadas con los rasgos de personalidad, las áreas corticales frontales están relacionadas con la iniciación y activación sexual (Arnow et al., 2002; Joyal, Black y Dassylva, 2007), siendo el funcionamiento neuropsicológico asociado con ellas un 
factor de relevancia en la explicación de la delincuencia sexual (Ward y Beech, 2006). Así diferentes trabajos relacionan la delincuencia sexual con una disfunción neurocognitiva frontal, basándose en que los agresores sexuales en general muestran un peor rendimiento en pruebas neuropsicológicas (como medidas de fluidez verbal, test de Stroop, Trail Making Test, torre de Londres, etc.) asociadas con el funcionamiento de estas áreas y circuitos frontales (Dolan, Millington y Park, 2002; Kelly, Richardson, Hunter y Knapp, 2002; Stone y Thompson, 2001).

A pesar de la evidente relación existente entre las bases neurobiológicas de los rasgos del MCF y las principales disfunciones neuropsicológicas de los agresores sexuales, pocos estudios han integrado ambos campos de investigación para examinar la relación existente entre funcionamiento neurocognitivo y rasgos normales de personalidad en estos delincuentes. Recientemente, Becerra-García, García-León y Egan (2013) estudiaron la posible relación entre el funcionamiento neurocognitivo frontal (evaluando capacidad y velocidad de procesamiento viso-motor y flexibilidad cognitiva, utilizando el Trail Making Test; TMT) y los rasgos de personalidad del MCF en agresores sexuales de menores. En este estudio se encontró que el rendimiento mostrado en las partes A y B del TMT se asociaban de manera independiente con los rasgos E y AP respectivamente (después de controlar variables de confusión relevantes como la edad, nivel educativo, historia de abuso de sustancias y presencia de psicopatología a lo largo de la vida).

Algunos autores proponen que el perfil de afectación neurocognitiva frontal es común a los delincuentes en general (Joyal et al., 2007) $\mathrm{y}$ que altas puntuaciones en el rasgo $\mathrm{N}$ y bajas en los rasgos AM y R están relacionadas con la agresión y la actividad criminal en general (Egan, 2011; Grumm y von Collani, 2009). Teniendo en cuenta esto, sería necesario clarificar si las relaciones entre el funcionamiento neurocognitivo y los rasgos normales de personalidad encontradas en el estudio de Becerra-García et al. (2013) se producen únicamente en agresores sexuales de menores o también se producen en otros grupos de delincuentes. Basándonos en esta investigación previa con agresores sexuales de menores, el presente trabajo pretende estudiar (mediante la misma metodología utilizada en dicho estudio previo) la relación entre los rasgos de personalidad y el funcionamiento neurocognitivo frontal en otros grupos de delincuentes y en una muestra control de participantes procedentes de la de población general. Concretamente, el trabajo tiene dos objetivos específicos. Primero, examinar si existe relación entre funcionamiento neurocognitivo frontal (capacidades de velocidad de procesamiento visomotora y la flexibilidad cognitiva) y rasgos de personalidad en los grupos de agresores sexuales de adultos, delincuentes no sexuales y control. Segundo, determinar si el rendimiento en las pruebas de funcionamiento neurocognitivo frontal (como el TMT) puede predecir algún porcentaje de la varianza en los dominios de personalidad del MCF. Este estudio nos ayudará a entender mejor los procesos neurocognitivos relacionados con la personalidad de los agresores sexuales y delincuentes no sexuales.

\section{Método}

\section{Participantes}

La muestra total estuvo compuesta por 99 participantes, todos hombres, con un rango de edad entre 21 y 66 años (media de edad de 39.13 años; DT = 11.47) y con el español como idioma nativo. Los participantes estaban incluidos en uno de los siguientes tres grupos: un grupo de delincuentes sexuales agresores de adultos, un grupo de delincuentes no sexuales y un grupo control procedente de la población general. Los participantes de ambos grupos de delincuentes estaban cumpliendo condena en diferentes prisiones españolas en el momento de la evaluación.

El grupo de delincuentes sexuales estaba formado por 26 personas (media de edad de 38.96 años; DT $=10.34$ ) condenadas por haber cometido delitos de agresión sexual contra adultos. Para este estudio, este tipo de delito 
fue definido como un contacto sexual no consentido con personas de 18 años o mayores. Este contacto sexual comprende manoseo de genitales o pechos sobre la ropa, así como los contactos sexuales "piel con piel», que incluían: los contactos mano-genitales, genitalesgenitales, boca-genital y genital-ano (Bourke y Hernández, 2009). En este grupo, un $84.6 \%$ $(\mathrm{n}=22)$ tenía un nivel educativo de primaria o inferior y el resto tenía un nivel de educación secundaría o superior. El $23.1 \%(\mathrm{n}=6)$ informaron de presencia de psicopatología a lo largo de su vida y un $61.5 \%(\mathrm{n}=16)$ informó sobre consumo de sustancias.

El grupo de delincuentes no sexuales estaba compuesto por un total de 31 participantes (edad media de 38.65; DT =10.64). Dentro de este grupo se incluían hombres condenados por delitos no sexuales (delitos de fraude, contra la propiedad, violencia de género, delitos por drogas, etc.). En este grupo, el 74.2 $\%(\mathrm{n}=23)$ informó tener un nivel educativo de primaria o inferior. Respecto a la presencia de psicopatología vital, un $35.5 \%(\mathrm{n}=11)$ de los participantes informo positivamente. Por su parte, un $61.3 \%(\mathrm{n}=19)$ manifestaron tener historia de consumo de sustancias.

Por último, el grupo control estuvo compuesto por hombres procedentes de la población general que no habían estado condenados previamente. Este grupo contó con un total de 42 participantes (con una media de 39.60 años de edad; DT $=10.98$ ). Las personas que formaron parte de este grupo fueron invitados a participar en el estudio. Educacionalmente, en el grupo control un $42.9 \%(n=18)$ informaron sobre un nivel de estudios primarios o inferior. Para este grupo la presencia de psicopatología y consumo de sustancias a lo largo de la vida fue de un $2.4 \%(\mathrm{n}=1)$ y de un $4.8 \%(\mathrm{n}=2)$, respectivamente.

\section{Instrumentos}

Inventario de Cinco Factores NEO (NEOFFI; NEO Five Factor Inventory; Costa y McCrae, 1992). El NEO-FFI es un cuestionario tipo autoinforme derivado del Inventario de
Personalidad NEO-Revisado (NEO PI-R, Revised NEO Personality Inventory; Costa y McCrae, 1992). Está compuesto por 60 ítem y proporciona una medida de cada uno de los cinco factores que componen el MCF de personalidad (N, E, AP, AM y R); cada uno de los elementos es valorado mediante la suma de las puntuaciones obtenidas en 12 ítem. La respuesta a cada ítem se proporciona en una escala tipo Likert de 5 puntos: "totalmente de acuerdo», "de acuerdo», «neutral», «en desacuerdo»y «totalmente en desacuerdo». En este estudio se utilizo la versión española del NEO-FFI (Costa y McCrae, 1999).

Trail Making Test (TMT; Reitan, 1992). El TMT evalúa diferentes habilidades neurocognitivas relacionadas con el funcionamiento de áreas frontales. La prueba consta de dos partes: la parte A es una medida de la capacidad y velocidad viso-motora y de procesamiento (Tirapu-Ustárroz y Luna-Lario, 2008), mientras que la parte $\mathrm{B}$ evalúa flexibilidad cognitiva (Kortte, Horner y Windham, 2002). La parte A (o TMT-A) consta de los números 1-25, cada uno de los cuales se encuentra dentro de un círculo, estando distribuidos de forma irregular en una hoja de papel. El objetivo es conectarlos en la secuencia correcta en el menor tiempo posible. La parte B (o TMT-B) requiere conectar números (1-13) y letras (A-L) en una secuencia alterna y ascendente (1-A-2-B-3-C, etc.) en el menor tiempo posible.

Entrevista sobre problemas psicopatológicos y uso de sustancias. Mediante esta entrevista breve utilizada en estudios previos (BecerraGarcía y García-León, 2011; Becerra-García et al., 2013), se recogía información acerca de la atención psiquiátrica y/o psicológica recibida a lo largo de su vida y del consumo de sustancias en cada uno de los participantes de los diferentes grupos. En primer lugar, a cada participante se le preguntaba si había recibido atención psiquiátrica-psicológica. Si la respuesta era afirmativa, se le preguntaba acerca de los síntomas que motivaron la intervención (ansiedad, depresión, trastornos psicóticos, trastornos de personalidad, trastornos sexuales). Por último se le interrogaba acerca del consumo (alcohol, 
hachís, cocaína, consumo múltiple) a lo largo de su vida.

\section{Procedimiento}

Antes de completar las medidas del estudio, a los participantes se les facilitó un documento de consentimiento informado. En este documento se les informaba que la investigación seguía las directrices éticas, que su participación era voluntaria y anónima y que podían abandonar la evaluación en cualquier momento sin ninguna consecuencia. Posteriormente, se recogían los datos relativos a edad y nivel educativo de cada participante y se administraba la entrevista sobre problemas psicopatológicos y uso de sustancias. Tras administrar esta entrevista se aplicaron de forma contrabalanceada el NEO-FFI y el TMT (partes A y B). Las diferentes medidas aplicadas se completaron en una sola sesión individual. Estas medidas fueron aplicadas a los diferentes grupos de delincuentes en las prisiones donde cumplían condena, mientras que los participantes del grupo control fueron evaluados en laboratorios de la universidad.

\section{Análisis estadístico}

La relación existente entre la puntuación en los diferentes dominios del MCF y el tiempo requerido para completar las partes A y B del TMT fue evaluada en cada grupo de participantes a través de una correlación bivariada de Pearson. Como varios grupos de participantes presentan un tamaño muestral cercano a 30, previamente se comprobaron en todos los grupos los supuestos necesarios para aplicar las pruebas paramétricas de correlación. Mediante el test $Z$ de Kolmogorov-Smirnov se comprobó que todas las variables se ajustan a la normalidad en los diferentes grupos. Para las diferentes medidas los valores $p$ para este test estaban comprendidos entre $p=.21$ (TMT parte A) y $p=.98$ (rasgo R) en el grupo de agresores sexuales de adultos, entre $p=.12$ (TMT parte B) y $p=.97$ (rasgo E) en el grupo de delincuentes no sexuales, $\mathrm{y}$ entre $p=.22$
(TMT parte A) y $p=.92($ TMT parte B) en el grupo control. Además, las variables son cuantitativas e independientes, otros dos criterios necesarios para realizar este tipo de análisis.

A partir de los datos obtenidos mediante el análisis de correlación de Pearson, y para determinar si el rendimiento en el TMT hace una contribución significativa e independiente a la predicción de varianza en los dominios del MCF, se realizo un análisis de regresión lineal múltiple (mediante el procedimiento stepwise) en cada uno de los grupos. Mediante este tipo de análisis se controlaron las diferentes variables de confusión en cada uno de los grupos (edad, nivel educativo, historia de abuso de sustancias y presencia de psicopatología a lo largo de la vida). Todos los análisis se realizaron usando el Statistical Package for the Social Sciences (SPSS) 17.0. El nivel de significación fue $p<.05$.

\section{Resultados}

El análisis de las relaciones entre los dominios de personalidad del MCF y las medidas de funcionamiento neurocognitivo evaluadas mediante el TMT mostró correlaciones significativas entre diferentes rasgos de personalidad y el tiempo requerido para completar el TMT en los grupos de delincuentes (ver tabla 1). No se hallaron correlaciones significativas entre las puntuaciones de los diferentes rasgos de personalidad y el rendimiento mostrado en el TMT por los participantes del grupo control.

En concreto, en el grupo de agresores sexuales de adultos se encontraron correlaciones negativas significativas entre el rasgo $\mathrm{E}$ y el tiempo necesario para completar la parte A del TMT, y entre el rasgo AM y el rendimiento mostrado también en la parte A del TMT. En el grupo de delincuentes no sexuales se encontró una correlación negativa significativa entre el rasgo $\mathrm{N}$ y el tiempo empleado en completar la parte A del TMT (ver tabla 1). La parte B del TMT no correlacionó significativamente con ninguno de los rasgos de personalidad evaluados en los diferentes grupos de participantes. 
Tabla 1.

Correlaciones entre los dominios del dominios del Modelo de los Cinco Factores y el Trail Making Test (partes A y B) en cada grupo.

\begin{tabular}{lcccccc}
\hline & \multicolumn{2}{c}{ Agresores sexuales } & \multicolumn{2}{c}{ Delitos no sexuales } & \multicolumn{2}{c}{ Grupo Control } \\
\cline { 2 - 7 } & TMT-A & TMT-B & TMT-A & TMT-B & TMT-A & TMT-B \\
\hline Neuroticismo. & -.22 & -.26 & $-.41^{*}$ & -.17 & .09 & .01 \\
Extraversión. & $-.42^{*}$ & -.32 & -.17 & -.07 & -.29 & -.29 \\
Apertura. & -.09 & .06 & -.32 & -.14 & -.07 & -.19 \\
Amabilidad. & $-.40^{*}$ & -.31 & -.06 & -.08 & .01 & .20 \\
Responsabilidad. & -.04 & .14 & -.06 & -.01 & .17 & .18 \\
\hline
\end{tabular}

Nota. TMT-A: tiempo empleado en la parte A del Trail Making Test; TMT-B: tiempo empleado en la parte B del Trail Making Test; * = $p<.05$

Se llevaron a cabo diferentes análisis de regresión lineal múltiple en los grupos donde se habían encontrado correlaciones bivariadas significativas entre medidas de personalidad y de funcionamiento neurocognitivo.

En el grupo de agresores sexuales de adultos se realizó un primer análisis de regresión lineal, incluyendo como variable dependiente el rasgo $\mathrm{E}$, e incluyendo como variables predictoras el rendimiento en el TMT (partes A y B) y las variables edad, nivel educativo, historia de psicopatología y consumo de sustancias. El tiempo requerido para completar la parte A del TMT fue introducido como única variable en el modelo, produciendo un cambio significativo en $R^{2}$, como puede observarse en la tabla 2. Este análisis también mostró que el tiempo requerido en completar la parte A del TMT es un predictor significativo de la varianza en el rasgo E en este grupo de delincuentes (como muestra el valor significativo del estadístico $t$, tabla 2). En el modelo los predictores eran independientes (factor de inflación de la varianza $(F I V)$ entre 1-1.21; $T$ entre .82-.99) y no existía autocorrelación entre los residuos (Durbin-Watson $=1.96)$. En este mismo grupo, un segundo análisis de regresión con el rasgo AM como variable dependiente, y con las mismas variables predictoras del análisis previo, no fue estadísticamente significativo $[F(1,24)=1.54 ; p=.22]$.

Por último, en el grupo de delincuentes no sexuales, se realizo un análisis de regresión en este caso con el $\mathrm{N}$ como variable dependiente y con las variables predictoras usadas anteriormente. En este análisis, el rendimiento en la parte A del TMT fue introducida como variable predictora en el primer paso $[F(1,29)=$ 5.95; $p=.02$ ] produciendo un cambio de $\mathrm{R}^{2}$ de .15. En el segundo paso fue introducida como variable predictora la presencia de psicopatología a lo largo de la vida (incrementando la $R^{2}$ en .12), conformando ambas variables el modelo predictivo para la varianza en el rasgo $\mathrm{N}$ que puede verse en la tabla 2 . En la tabla 2 también se observa que ambas variables se relacionan significativamente con el N. En este modelo los predictores también eran independientes ( $F I V$ entre 1-1.15; $T$ entre .87-.99) y no existía autocorrelación entre los residuos (Durbin-Watson $=2.1$ ).

Tabla 2.

Influencia del rendimiento en el Trail Making Test en los dominios del Modelo de los Cinco Factores en agresores sexuales de adultos y delincuentes no sexuales. Análisis de regresión significativos.

\begin{tabular}{llcccc}
\hline \multicolumn{7}{c}{ Agresores sexuales de adultos } \\
\hline Dependiente. & $R^{2 .}$ & Predictores & Modelo de Regresión & $\beta$. & $t$. \\
Extraversión. & .17 & TMT-A & $F(1,24)=4.47^{*}$. & -.41 & $-2.11^{*}$ \\
\hline
\end{tabular}




\begin{tabular}{lccccc}
\hline \multicolumn{7}{c}{ Delincuentes no sexuales } \\
\hline Dependiente. & $R^{2 .}$ & Predictores & Modelo de Regresión & $\beta$ & $t$ \\
Neuroticismo. & .27 & TMT-A & $F(1,29)=6.54^{*}$ & -.44 & $-2.83^{*}$ \\
& & Psicopatología & & -.38 & $2.46^{*}$ \\
\hline
\end{tabular}

Nota. Psicopatología: presencia de psicopatología a lo largo de la vida; $R^{2}$ : varianza explicada; TMT-A: tiempo empleado en la parte A del Trail Making Test; * $=p<.05$.

\section{Discusión}

El presente estudio evidencia que el rendimiento mostrado en la parte A del TMT se relaciona con diferentes rasgos de personalidad del MCF en delincuentes. En agresores sexuales de adultos, la parte A del TMT muestra una correlación negativa con el rasgo E, mientras que el rendimiento en dicha parte A del TMT se relaciona también de manera significativa y negativa con el $\mathrm{N}$ en agresores no sexuales. Los resultados muestran que: a) en agresores sexuales de adultos, un peor rendimiento (mayor tiempo empleado) en la parte A del TMT se relaciona con una menor puntuación en el rasgo $\mathrm{E}$, o bien que un buen rendimiento en la parte A del TMT se relaciona con una elevada puntuación en $\mathrm{E} ; \mathrm{y}$ b) en delincuentes no sexuales, un rendimiento más bajo en la parte A del TMT se relaciona con menor $\mathrm{N}$, o que un buen rendimiento en esta parte A del TMT se relaciona con un mayor nivel de N. Estas asociaciones halladas entre el tiempo empleado en la parte A del TMT y los rasgos $\mathrm{E}$ y $\mathrm{N}$ en agresores sexuales de adultos y delincuentes no sexuales, respectivamente, se mantienen significativas cuando se controlan otras variables de confusión, lo que indica que el rendimiento en esta parte A del TMT se relaciona independientemente con estos rasgos de personalidad en ambos grupos.

Las medidas psicométricas de capacidad viso-motora y velocidad de procesamiento correlacionan con el rendimiento en diferentes habilidades cognitivas e intelectuales ( $\mathrm{Li}$ et al., 2004; Salthouse, 2005). El presente estudio muestra que en población reclusa la capacidad viso-motora y velocidad de procesamiento, evaluados mediante el TMT parte A, también correlacionan con medidas de personalidad. En el caso de agresores sexuales de adultos, se observa una relación negativa entre la velocidad de procesamiento viso-motor y el rasgo E similar a la obtenida en estudios previos con agresores de menores (Becerra-García et al., 2013). En general los agresores sexuales son más introvertidos que los delincuentes no sexuales y participantes controles sin antecedentes penales (Becerra-García, García-León Muela-Martínez y Egan, 2013), por lo que su rendimiento es peor en la parte A del TMT. Lo hallado en este trabajo junto con los resultados de investigaciones previas apunta, al menos de manera preliminar, a que esta relación entre la capacidadvelocidad de procesamiento viso-motor con la dimensión E parece ser característica de los agresores sexuales independientemente de la edad de la víctima, además de ser un hallazgo parecido a los que muestran recientes estudios realizados con muestras clínicas (enfermedades neurodegenerativas que afectan a áreas y conexiones neurales fronto-temporales), los cuales evidencian una disminución significativa en el rasgo de personalidad $\mathrm{E}$ asociada con alteración de las capacidades ejecutivas (Mahoney, Rohrer, Omar, Rossor y Warren, 2011; Sollberger et al., 2011), entre las que se encuentra la capacidad-velocidad de procesamiento. Estos grupos clínicos también muestran síntomas conductuales similares a los mostrados por agresores sexuales, como el retraimiento social y la conducta sexual desinhibida (inapropiada e indiscriminada), además de conductas de agresión o robo patológico (Méndez, Shapira y Saul, 2011; Poetter y Stewart, 2012).

En cuanto a los delincuentes no sexuales existe mejor rendimiento en la parte A del TMT si el nivel de $\mathrm{N}$ es mayor. Estos resultados confirman que en este grupo una elevada puntuación en el rasgo $\mathrm{N}$ no afecta negativamente al rendimiento en esta parte del TMT, estando en consonancia estos resultados con lo obtenido por diferentes trabajos realizados en población no clínica (Ayotte, Potter, Williams, Ste- 
ffens y Bosworth, 2009; Jelicic et al., 2003) y con muestras clínicas donde el déficit cognitivo más acentuado es la velocidad de procesamiento (Akbar, Honarmand y Feinstein, 2011).

En el grupo de control de nuestro estudio no se ha encontrado ninguna asociación significativa entre el rendimiento neurocognitivo y los rasgos de personalidad, aunque los datos parecen apuntar en la misma dirección descrita entre la E y la parte A del TMT para los agresores sexuales. A pesar de que este aspecto tiene que ser estudiado y confirmado en otras investigaciones, con muestras distintas y suficientemente amplias, lo obtenido es este trabajo y en estudios previos podrían sugerir que la capacidad-velocidad de procesamiento viso-motor y la E podrían relacionarse de modo similar en agresores sexuales y muestras clínicas con daño neurológico, pudiendo ser la existencia de disfunción neural una variable de importancia para que los sujetos más introvertidos tengan también peor velocidad de procesamiento y capacidad viso-motriz. Este motivo, tal vez podría explicar porque la relación entre ambas medidas es más fuerte y significativa en el grupo de agresores sexuales, a pesar de ser el que cuenta con menor tamaño muestral.

En cuanto al segundo objetivo del trabajo, se puede afirmar que el rendimiento mostrado en la parte A del TMT contribuye a la predicción de un porcentaje significativo de la varianza en los rasgos de personalidad $\mathrm{E}$ y $\mathrm{N}$ en las muestras de agresores sexuales y de delincuentes no sexuales respectivamente. Desde una perspectiva clínica, el rendimiento en la parte A del TMT nos informa de forma directa sobre la capacidad-velocidad de procesamiento viso-motriz del delincuente evaluado. Además, por los resultados obtenidos, la ejecución de la parte A del TMT podría ser un «indicio indirecto» de cómo podría puntuar el delincuente sexual-no sexual en los dominios E y N (si tenemos en cuenta el porcentaje de varianza que explica de ambos dominios), el cual debe ser confirmado mediante una correcta evaluación de los diferentes rasgos que conforman cada dominios de personalidad.

Este estudio supera algunas de las limitaciones del estudio que realizamos previamente con agresores sexuales de menores (BecerraGarcía et al., 2013), y muestra la existencia de semejanzas entre diferentes grupos de delincuentes sexuales. Como principal limitación del trabajo, se puede destacar la relativa al número de participantes incluidos. El pequeño tamaño muestral de cada grupo hace que tengamos que interpretar estos datos como preliminares, a pesar de que los resultados estén en la misma línea que lo obtenido por otros estudios con mayor potencia estadística y de realizar un control exhaustivo de los requisitos necesarios para utilizar el análisis estadístico empleado. Por otro lado, es posible que los resultados se vean afectados por la influencia general del cociente intelectual. Futuras investigaciones deberían superar estas limitaciones incluyendo mayor número de participantes y nuevas variables de control. En estos futuros trabajos podría ser útil incluir diferentes medidas para examinar si otras capacidades ejecutivas (como por ejemplo, planificación, toma de decisiones, inhibición, componentes multitarea, etc.) están relacionadas con los rasgos de personalidad en muestra forense.

En conclusión, la relación entre el funcionamiento neurocognitivo (evaluado mediante el TMT) y la personalidad (examinada desde el modelo de los Cinco Factores) parece ser diferente en función del tipo de delito cometido (sexual-no sexual), aunque los resultados deben ser interpretados de forma preliminar debido a las limitaciones del trabajo y a la necesidad que se realicen investigaciones con mayor número de participantes que lo confirmen.

\section{Referencias}

Akbar, N., Honarmand, K. y Feinstein, A. (2011). Self-assessment of cognition in multiple sclerosis: the role of personality and anxiety. Cognitive and Behavioral Neurology, 24, 115-121. http:// dx.doi.org/10.1097/WNN.0b013e31822a20ae

Arnow, B. A., Desmond, J. E., Banner, L. L., Glover, G. H., Solomon, A., Polan, M. L., Lue, T. F. y Atlas, S. W. (2002). Brain activation and sexual arousal in healthy, heterosexual males. Brain, 125, 10141023. http://dx.doi.org/10.1093/brain/awf108 
Ayotte, B. J., Potter, G. G., Williams, H. T., Steffens, D. C. y Bosworth, H. B. (2009). The moderating role of personality factors in the relationship between depression and neuropsychological functioning among older adults. International Journal of Geriatric Psychiatry, 24, 1010-1019. http://dx.doi.org/10.1002/gps.2213

Becerra-García, J. A. y García-León, A. (2011). Does a childhood abuse history influence choice of the sex of victims of sex aggressors? Romanian Journal of Legal Medicine, 19, 295-298. http:// dx.doi.org/10.4323/rjlm.2011.295

Becerra-García, J. A., García-León, A. y Egan, V. (2013). Toward a neuropsychology of personality in sex offenders against children: an exploratory psychometric study. Journal of Child Sexual Abuse, 22, 612-623. http://dx.doi.org/10.1 080/10538712.2013.800933

Becerra-García, J. A., García-León, A., Muela-Martínez, J. A. y Egan, V. (2013). A controlled study of the Big Five personality dimensions in sex offenders, non-sex offenders and non-offenders: relationship with offending behaviour and childhood abuse. Journal of Forensic Psychiatry and Psychology, 24, 233-246. http://dx.doi.org/10 $.1080 / 14789949.2013 .764463$

Bourke, M. L. y Hernández, A. E. (2009). The 'Butner Study' redux: a report of the incidence of handson child victimization by child pornography offenders. Journal of Family Violence, 24, 183-191. http://dx.doi.org/10.1007/s10896-008-9219-y

Charlton, R. A., Barrick, T. R., Markus, H. S. y Morris, R. G. (2009). Theory of mind associations with other cognitive functions and brain imaging in normal aging. Psychology and Aging, 24, 338-348. http://dx.doi.org/10.1037/a0015225

Cohen, M. X., Schoene-Bake, J. C., Elger, C. E. y Weber, B. (2009). Connectivity-based segregation of the human striatum predicts personality characteristics. Nature Neuroscience, 12, 32-34. http://dx.doi.org/10.1038/nn.2228

Costa, P. T. y McCrae, R. R. (1992). Revised NEO personality inventory (NEO-PI-R) and NEO fivefactor inventory (NEO-FFI) professional manual. Odessa: Psychological Assessment Resources.

Costa, P. T. y McCrae, R. R. (1999). NEO PI-R, Inventario de Personalidad NEO Revisado. NEOFFI, Inventario NEO reducido de Cinco Factores. Manual. Madrid: TEA Ediciones.
Cremers, H. R., Demenescu, L. R., Aleman, A., Renken, R., van Tol, M. J., van der Wee, N., Veltman, D. J. y Roelofs, K. (2010). Neuroticism modulates amygdale-prefrontal connectivity in response to negative emotional facial expressions. NeuroImage, 49, 963-970. http://dx.doi. org/10.1016/j.neuroimage.2009.08.023

Delano-Wood, L., Abeles, N., Sacco, J. M., Wierenga, C. E., Horne, N. R. y Bozoki, A. (2008). Regional white matter pathology in mild cognitive impairment: differential influence of lesion type on neuropsychological functioning. Stroke, 39, 794-799. http://dx.doi.org/10.1161/ STROKEAHA.107.502534

De Young, C. G., Hirsh, J. B., Shane, M. S., Papademetris, X., Rajeevan, N. y Gray, J. R. (2010). Testing predictions from personality neuroscience: brain structure and the big five. Psychological Science, 21, 820-828. http://dx.doi. org/10.1177/0956797610370159

Dolan, M., Millington, J. y Park, I. (2002). Personality and neuropsychological function in violent, sexual, and arson offenders. Medicine, Science and Law, 42, 34-43.

Egan, V. (2011). Individual differences and antisocial behaviour. En A. Furnham, S. von Stumm y K. Petredies (Eds.), Handbook of individual differences (pp. 512-537). Oxford: Blackwell-Wiley.

Grumm, M. y von Collani, G. (2009). Personality types and self-reported aggressiveness. Personality and Individual Differences, 47, 845-850. http://dx.doi.org/10.1016/j.paid.2009.07.001

Jackson, J., Balota, D. A. y Head, D. (2011). Exploring the relationship between personality and regional brain volume in healthy aging. Neurobiology of Aging, 32, 2162-2171. http://dx.doi. org/10.1016/j.neurobiolaging.2009.12.009

Jelicic, M., Bosma, H., Ponds, R. W., Van Boxtel, M. P., Houx, P. J. y Jolles, J. (2003). Neuroticism does not affect cognitive functioning in later life. Experimental Aging Research, 29, 73-78. http://dx.doi.org/10.1080/03610730303704

Joyal, C. C., Black, D. N. y Dassylva, B. (2007). The neuropsychology and neurology of sexual deviance: a review and pilot study. Sexual Abuse: A Journal of Research and Treatment, 19, 155-173. http://dx.doi.org/10.1177/107906320701900206 
Kelly, T., Richardson, G., Hunter, R. y Knapp, M. (2002). Attention and executive function deficits in adolescent sex offenders, Child Neuropsychology, 8, 138-143.

Kortte, K. B., Horner, M. D. y Windham, W. K. (2002). The trail making test, part B: Cognitive flexibility or ability to maintain set? $A p$ plied Neuropsychology, 9, 106-109. http://dx.doi. org/10.1207/S15324826AN0902_5

Li, S. C., Lindenberger, U., Hommel, B., Aschersleben, G., Prinz, W. y Baltes, P. B. (2004). Transformations in the couplings among intellectual abilities and constituent cognitive processes across the life span. Psychological Science, 15, 155-163. http://dx.doi.org/10.1111/ j.0956-7976.2004.01503003.x

Mahoney, C. J., Rohrer, J. D., Omar, R., Rossor, M. N. y Warren, J. D. (2011). Neuroanatomical profiles of personality change in frontotemporal lobar degeneration. British Journal of Psychiatry, 198, 365-372. http://dx.doi.org/10.1192/bjp. bp. 110.082677

McCrae, R. R. y Costa, P. T. (1997). Personality trait structure as a human universal. American Psychologist, 52, 509-516. http://dx.doi. org/10.1037/0003-066X.52.5.509

Mendez, M. F., Shapira, J. S. y Saul, R. E. (2011). The spectrum of sociopathy in dementia. Journal of Neuropsychiatry and Clinical Neurosciences, 23, 132-140. http://dx.doi.org/10.1176/ appi.neuropsych.23.2.132

Nettle, D. y Liddle, B. (2008). Agreeableness is related to social-cognitive, but not socialperceptual, theory of mind. European Journal of Personality, 22, 323-335. http://dx.doi.org/10.1002/per.672

Poetter, C. E. y Stewart, J. T. (2012). Treatment of indiscriminate, inappropriate sexual behavior in frontotemporal dementia with carbamazepine. Journal of Clinical Psychopharmacology, 32, 137-138. http://dx.doi.org/10.1097/ JCP.0b013e31823f91b9

Reitan, R. M. (1992). Trail making test: Manual for administration and scoring. Tucson: Reitan Neuropsychology Laboratory.

Salthouse, T. A. (2005). Relations between cognitive abilities and measures of executive functioning. Neuropsychology, 19, 532-545. http://dx.doi. org/10.1037/0894-4105.19.4.532
Schretlen, D. J., van der Hulst, E. J., Pearlson, G. D. y Gordon, B. (2010). A neuropsychological study of personality: trait openness in relation to intelligence, fluency, and executive functioning. Journal of Clinical and Experimental Neuropsychology, 32, 1068-1073. http://dx.doi. org/10.1080/13803391003689770

Sollberger, M., Neuhaus, J., Ketelle, R., Growdon, M., Jung, J., Miller, B. L. y Rankin, K. P. (2011). Interpersonal traits change as a function of disease type and severity in neurodegenerative disease. Journal of Neurology, Neurosurgery and Psychiatry, 82, 732-739. http://dx.doi. org/10.1136/jnnp.2010.205047

Stone, M. H. y Thompson, E. H. (2001). Executive function impairment in sexual offenders. Journal of Individual Psychology, 57, 51-59.

Takeuchi, H., Taki, Y., Sassa, Y., Hashizume, H., Sekiguchi, A., Fukushima, A. y Kawashima, R. (2010). White matter structures associated with creativity: evidence from diffusion tensor imaging. NeuroImage, 51, 11-18. http://dx.doi. org/10.1016/j.neuroimage.2010.02.035

Weschler, D. (2001). WAIS-III. Escala de inteligencia Wechsler para adultos. Tercera versión. Manual de aplicación y corrección. Madrid: TEA ediciones.

Tirapu-Ustárroz, J. y Luna-Lario, P. (2008). Neuropsicología de las funciones ejecutivas. En J. Tirapu-Ustárroz, M. Ríos-Lago y F. Maestú (Eds.), Manual de neuropsicología (pp. 219-249). Barcelona: Viguera Editores.

Turken, A. U., Whitfield-Gabrieli, S., Bammer, R., Baldo, J., Dronkers, N. y Gabrieli, J. (2008). Cognitive processing speed and the structure of white matter pathways: Convergent evidence from normal variation and lesion studies. NeuroImage, 42, 1032-1044. http://dx.doi. org/10.1016/j.neuroimage.2008.03.057

Ward, T. y Beech, A. (2006). An integrated theory of sexual offending. Aggression and Violent Behavior, 11, 44-63. http://dx.doi.org/10.1016/j. avb.2005.05.002

Xu, J. y Potenza, M. N. (2012). White matter integrity and five-factor personality measures in healthy adults. NeuroImage, 59, 800-807. http:// dx.doi.org/10.1016/j.neuroimage.2011.07.040 